PROCEEDINGS OF THE

AMERICAN MATHEMATICAL SOCIETY

Volume 139, Number 2, February 2011, Pages 757-768

S 0002-9939(2010)10664-0

Article electronically published on September 27, 2010

\title{
ON THE SPECTRUM OF $(\operatorname{Spin}(10,2), S L(2, \mathbb{R}))$ IN $E_{7,3}$
}

\author{
ZHE DU
}

(Communicated by Kathrin Bringmann)

\begin{abstract}
We give the complete description of the spectrum of $(\operatorname{Spin}(10,2), S L(2, \mathbb{R}))$ in the minimal representation of $E_{7,3}$, and we find that it only consists of a discrete spectrum. This is interesting as both groups are noncompact. Also, most of the representations occurring in the spectrum are unitary representations with nonzero cohomology. The representations we obtained have different Hodge types from the representations we obtain from classical theta liftings.
\end{abstract}

\section{INTRODUCTION}

The dual pair theory has been well understood in the symplectic case. Suppose $\left(G, G^{\prime}\right)$ is a dual pair inside $S p_{2 n}(\mathbb{R})$; i.e., both groups are reductive subgroups of $S p_{2 n}(\mathbb{R})$ and they are centralizers of each other. Let $\tilde{S} p$ be the metaplectic two-fold cover of $S p$. For any subgroup $E$ of $S p$, let $\tilde{E}$ denote its inverse image in $\tilde{S} p$. Then it is well known that $\left(\tilde{G}, \tilde{G}^{\prime}\right)$ are centralizers of each other inside $\tilde{S} p$. By the Stone-Von Neumann theorem, for each choice of a nontrivial additive character, there exists an irreducible unitary representation $\pi$ for $\tilde{S} p$, which we call the Weil representation. The restriction of $\pi$ to the dual pair $\left(\tilde{G}, \tilde{G}^{\prime}\right)$ gives a one-to-one correspondence between certain representations of these two groups. This result had been proved for the Archimedean case and the $p$-adic case for $p \neq 2$. For details, see e.g. [4], 6], [7, [13.

It is natural to consider other groups different from symplectic groups, find similar representations to the Weil representation and investigate the correspondence. We require the representation to be as small as possible but not trivial: it has minimal Gelfand-Kirillov dimension and is associated with the minimal coadjoint orbit. We call such a representation the minimal representation. For details, see [26].

Let $E_{7,3}$ be the connected Lie group of real rank 3 and type $E_{7}$. There is a minimal representation $\pi_{\min }$ which is an irreducible unitary representation of $E_{7,3}$ with minimal Gelfand-Kirillov dimension, and whose annihilator in the (complexified) universal enveloping algebra is the Joseph ideal.

In this paper, we will give a complete description of the spectrum of the restriction of $\pi_{\min }$ to the subgroup $\operatorname{Spin}(10,2) \times S L(2, \mathbb{R})$. It turns out that the discrete spectrum gives the full description of the restriction. As both of the two groups are noncompact, it is interesting that the full spectrum consists of only a discrete

Received by the editors March 28, 2010.

2000 Mathematics Subject Classification. Primary 22E46.

(C)2010 American Mathematical Society Reverts to public domain 28 years from publication 
spectrum. For an integer $k \geq 2$, let $\pi_{-k}$ be the antiholomorphic discrete series representation of $S L(2, \mathbb{R})$ of highest weight $-k$. Write the unitary decomposition of $\pi_{\min }$ as

$$
\left.\pi_{\min }\right|_{S p i n(10,2) \times S L(2, \mathbb{R})}=\bigoplus_{k \geq 4} \theta_{k} \otimes \pi_{-k} .
$$

We will describe $\theta_{k}$ for $k \geq 4$ in section 4 . Also, we can see that $\theta_{k}$ is a highest weight module. For $k \geq 4$, the $K$-type structure of $\theta_{k}$ is identical to that of a derived functor module. For $k \geq 6, \theta_{k}$ is an irreducible unitary representation with nonzero cohomology.

To determine $\theta_{k}$ we use the fact that $\operatorname{Spin}(10,2)$ and $S L(2, \mathbb{R})$ form a reductive dual pair in $E_{7,3}$ corresponding to the $\alpha$-case as in 23 .

It should be mentioned that Kobayashi 22 has studied branching laws with similar situations, namely an irreducible unitary highest weight representation of a Lie group restricted to a reductive symmetric subgroup consisting of a discrete spectrum. However, our results gives the explicit description which has important applications in automorphic forms and Shimura varieties.

As $(\operatorname{Spin}(10,2), S L(2, \mathbb{R}))$ is also a dual pair inside $S p_{24}(\mathbb{R})$, we have classical theta liftings in [17]. From [10, we see that unitary representations with nonzero cohomology of $\operatorname{Spin}(10,2)$ can occur with certain Hodge degrees. The Hodge degrees of representations from classical theta liftings with dual pair $\left(O(10,2), S p_{2 n}\right)(n \leq 5)$ are $(r, r)$ with $0 \leq r \leq 10$. Our representations have degree $(5,0)$ and $(0,5)$. So we have produced representations that cannot be obtained from classical liftings.

We will use the following method to determine the spectrum.

Let $K$ be the maximal compact subgroup of $E_{7,3}$ and $K_{1}=\operatorname{Spin}(10) \times S O(2)$ the maximal compact subgroup of $\operatorname{Spin}(10,2)$. We first restrict $\pi_{\min }$ to $\operatorname{Spin}(10) \times$ $S O(2) \times S O(2)$, where the decomposition takes the form

$$
\left.\pi_{\min }\right|_{\operatorname{Spin}(10) \times S O(2) \times S O(2)}=\bigoplus_{p, q, r \geq 0} \pi\left(p \omega_{6}+q \omega_{2}\right) \times\left(\mu_{1}\right) \times\left(\mu_{2}\right),
$$

where $\omega_{j}$ denotes the $j$-th fundamental weight for $\operatorname{Spin}(10), \pi(\lambda)$ is the irreducible finite dimensional representation with highest weight $\lambda$, and $\mu_{1}, \mu_{2}$ are one dimensional representations of $S O(2)$ related to $p, q, r$. One knows (see [11]) that $\theta_{k}$ is quasi-simple and its infinitesimal character is determined by the corresponding representation of $S L(2, \mathbb{R})$. Together with the $K$-types we will have enough information to give the description of the representation.

We will generalize our results to global fields and talk about their applications to automorphic forms and Shimura varieties in the near future.

\section{EMBEDDING}

We first recall the structure of $E_{7,3}$ and set up some notation. Consider the group $G=E_{7,3}$, a connected exceptional Lie group with split real rank 3 . Its maximal compact subgroup is $K=E_{6} \times T$, where $T$ is a torus of $E_{7,3}$ and $E_{6}$ is a compact subgroup of type $E_{6}$. $K$ contains a compact Cartan subgroup $H$, and $E_{7,3}$ is of Hermitian type. Let $\mathfrak{e}_{7,3}, \mathfrak{e}_{6,0}$ and $\mathfrak{t}(\mathfrak{e})_{7,3}$ be the corresponding complexified Lie algebra of $E_{7,3}, E_{6}$ and $T$ respectively. We introduce coordinates so that the complexified Lie algebra $\mathfrak{h}$ of $H$ lies in $\mathbb{C}^{8}$ and such that the restriction of the Cartan-Killing form is the standard inner product given by

$$
\langle x, y\rangle=x_{1} y_{1}+\ldots+x_{8} y_{8} .
$$


Let $e_{j}$ denote the evaluation on the $j$-th coordinate. We will use the same notation as in Bourbaki [1, and the roots are as follows:

$$
\pm e_{i} \pm e_{j},(1 \leq i<j \leq 6), \pm\left(e_{8}-e_{7}\right),
$$

and

$$
\pm \frac{1}{2}\left(e_{8}-e_{7}+\sum_{1 \leq i \leq 6}(-1)^{\nu_{i}} e_{i}\right) \quad \text { with } \quad \Sigma_{1 \leq i \leq 6} \nu_{i} \quad \text { odd }
$$

The simple roots are

$$
\begin{gathered}
\alpha_{1}=\frac{1}{2}\left(e_{8}-e_{7}-e_{6}-e_{5}-e_{4}-e_{3}-e_{2}+e_{1}\right), \\
\alpha_{2}=e_{2}+e_{1}, \\
\alpha_{i}=e_{i-1}-e_{i-2}(3 \leq i \leq 7) .
\end{gathered}
$$

The half sum of positive roots is

$$
\rho=\left(0,1,2,3,4,5,-\frac{17}{2}, \frac{17}{2}\right) .
$$

We know the simple roots for the Lie group $E_{7,3}$, with fundamental Cartan subgroup, are simple roots with $\alpha_{7}$ noncompact and the other roots compact. The Vogan diagram is

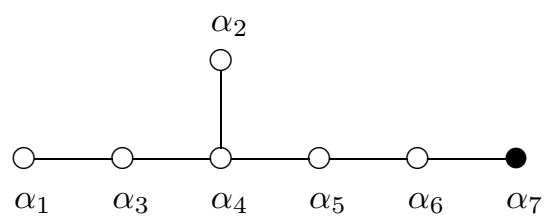

Here a painted point means a noncompact simple root and an unpainted point means a compact simple root.

The half sum of compact roots is

$$
\rho_{c}=(0,1,2,3,4,-4,-4,4) .
$$

With the above notation, $K=E_{6} \times T$, where $E_{6}$ corresponds to the compact roots.

Fix an orientation for the torus $T$ by $t_{7,3}=(0,0,0,0,0,2,-1,1) \in \mathfrak{t}\left(\mathfrak{e}_{7,3}\right) \subset \mathfrak{h}$. The Cartan decomposition of $\mathfrak{e}_{7,3}$ is

$$
\mathfrak{e}_{7,3}=\mathfrak{e}_{6,0} \oplus \mathfrak{t}\left(\mathfrak{e}_{7,3}\right) \oplus \mathfrak{p}^{+} \oplus \mathfrak{p}^{-},
$$

where as $\left(\mathfrak{e}_{6,0} \oplus \mathfrak{t}_{7,3}\right)$-modules, $\mathfrak{p}^{+}=V\left(\omega_{1}\right) \times(2)_{7,3}, \mathfrak{p}^{-}=V\left(\omega_{6}\right) \times(-2)_{7,3}$. The highest weights for $\mathfrak{p}^{+}, \mathfrak{p}^{-}$are $\omega_{1}=e_{8}-e_{7}$ and $\omega_{6}=e_{5}-e_{6}$ respectively. Here $\omega_{1}, \omega_{6}$ are the fundamental weights corresponding to $\alpha_{1}, \alpha_{6}$ respectively and $( \pm 2)_{7,3}$ denotes the action of $t_{7,3}$ on $\mathfrak{p}^{ \pm}$.

Let $\beta=e_{8}-e_{7}$ be the highest root of $E_{7,3}$ which is noncompact. Then in the extended Dynkin diagram, $-\beta$ will be linked to $\alpha_{1}$ and is disjoint from the others. Deleting $\alpha_{1}$ from the extended Dynkin diagram, we can get two disjoint parts. One part corresponds to a noncompact root identified with $S L(2, \mathbb{R})$, and the other part corresponds to the Lie algebra of $\operatorname{Spin}(10,2)$. With this identification, we get the embedding of $\operatorname{Spin}(10,2) \times S L(2, \mathbb{R})$ into $E_{7,3}$. 
Consider $U=K \cap(\operatorname{Spin}(10,2) \times S L(2, \mathbb{R}))$. From the above identification we know that

$$
U=\operatorname{Spin}(10) \times S O(2)_{1} \times S O(2)_{2},
$$

where $\operatorname{Spin}(10) \times S O(2)_{1}$ is the maximal compact subgroup of $\operatorname{Spin}(10,2)$ and $S O(2)_{2}$ is the maximal compact subgroup of $S L(2, \mathbb{R})$ corresponding to the noncompact root $\left(e_{7}-e_{8}\right)$. We denote the Lie algebra of $\operatorname{Spin}(10,2)$ by $\mathfrak{s o}(10,2)$ and the Lie algebra of $\operatorname{Spin}(10)$ by $\mathfrak{s o}(10)$.

We will use the above notation without further explanation in the latter part of this paper.

\section{RESTRICTION}

Let $\pi_{\min }$ be the minimal representation of $E_{7,3}$. Noting that $E_{7,3}$ is of Hermitian type, Enright, Howe, and Wallach [3] have given a classification of highest weight modules for all Hermitian symmetric cases. So we can read the $K$-types:

$$
\pi_{\text {min }} \mid K=\bigoplus_{n \geq 0} V(n \lambda) \otimes \mathbb{C}_{-4 \xi} .
$$

Here $\lambda=e_{5}-e_{6}$ and $\xi=e_{6}+\frac{1}{2}\left(e_{8}-e_{7}\right) . V(n \lambda)$ is the highest weight module of $K$ with highest weight $n \lambda$, and $\mathbb{C}_{-4 \xi}$ is the one dimensional representation of $K$ with weight $-4 \xi$,

With this information we know the highest weight representation in the classification of Enright, Howe, and Wallach 3 with given $K$-types has Gelfand-Kirillov dimension of 17, which is indeed the minimal Gelfand-Kirillov dimension. For the computation of the dimension, we can use Theorem 1.2 in 24. The minimal dimension of the coadjoint orbit can be found in [2]. The computations are as follows.

Theorem 3.1 (Vogan). Suppose that $\pi$ is an irreducible admissible representation of $G$. Let $X$ be the $K$-finite vectors in $\pi$, $d$ the Gelfand-Kirillov dimension of $X$, and $N_{X}(t)$ the sum of the dimensions of the eigenspaces of $\Omega_{K}$ with eigenvalues less than or equal to $t^{2}$. Here $K$ is a maximal compact subgroup of $G$ and $\Omega_{K}$ is the Casimir operator of $K$. Then there is a constant $A>0$, depending on $X$, such that for $t \geq 1$,

$$
A^{-1} t^{d} \leq N_{X}(t) \leq A t^{d}
$$

Proof. See [24.

Proposition 3.1. The Gelfand-Kirillov dimension of $\pi_{\min }$ is 17 , and it has the minimal Gelfand-Kirillov dimension.

Proof. We know that the $K$-type decomposition of $\pi_{\min }$ is

$$
\pi_{\text {min }} \mid K=X=\bigoplus V(n \lambda) \otimes \mathbb{C}_{-4 \xi}
$$

Write $F_{n \lambda-4 \xi}=V(n \lambda) \otimes \mathbb{C}_{-4 \xi}$. Using the Weyl dimension formula, we find that

$$
\operatorname{dim} F_{n \lambda-4 \xi}=c_{16} n^{16}+c_{15} t^{15}+\ldots+c_{0},
$$

where the $c_{i}$ are constants.

The action of $\Omega_{K}$ on $F_{n \lambda-4 \xi}$ is

$$
\left\|n \lambda-4 \xi+\rho_{c}\right\|^{2}-\left\|\rho_{c}\right\|^{2}=2 n^{2}+24 n+24 .
$$

Here $\rho_{c}=(0,1,2,3,4,-4,-4,4)$ and $\rho_{c}+n \lambda-4 \xi=(0,1,2,3, n+4,-n-8,-2,2)$. 
We know that

$$
N_{X}(t)=\sum_{2 n^{2}+24 n+24 \leq t^{2}} \operatorname{dim} F_{n \lambda-4 \xi}
$$

This is equivalent to

$$
\sum_{n \leq \frac{t}{\sqrt{2}}}\left(c_{16} n^{16}+c_{15} n^{15}+\ldots+c_{0}\right)
$$

when $t$ is sufficiently large. Considering $\int_{1}^{m} t^{l} d t=O\left(m^{l+1}\right)$, we know that $1^{l}+$ $2^{l}+\ldots+m^{l}=O\left(m^{l+1}\right)$. So $N_{X}(t)=O\left(t^{17}\right)$. By Theorem 3.1, the Gelfand-Kirillov dimension of $\pi_{\min }$ is 17 .

We will use the following results in the proof of the restriction. For details, see [11.

Let $K$ be a compact connected Lie group with Cartan subgroup $T$. Let $K_{1} \subseteq K$ be a closed connected subgroup with Cartan subgroup $T_{1} \subseteq T$. Fix compatible Weyl chambers for the pairs $(K, T)$ and $\left(K_{1}, T_{1}\right)$. Let $\psi \in \hat{T}$ be a dominant weight, $V_{n \psi}$ be the irreducible $K$-module with highest weight $n \psi$ and

$$
\left.V_{\psi}\right|_{K_{1}}=\bigoplus_{j=1}^{r} W_{\phi_{j}}
$$

be a decomposition of the restriction of $V_{\psi}$ to $K_{1}$, where $W_{\psi_{j}}$ is an irreducible $K_{1}$ module with highest weight $\phi_{j}$. Note that $V_{n \psi} \subseteq V_{\psi}{ }^{\otimes n}$. It is obvious that $V_{\psi}{ }^{\otimes n}$ contains all irreducible $K_{1}$-modules with highest weights of the form

$$
k_{1} \phi_{1}+\ldots+k_{r} \phi_{r}, \quad k_{1}+\ldots+k_{r}=n,
$$

where the $k_{j}$ 's are nonnegative integers. The Borel-Weil theorem implies the following.

Lemma 3.2. In the above setting, the restriction of $V_{n \psi}$ to $K_{1}$ contains all the irreducible $K_{1}$-modules with highest weights of the form (3.1).

Lemma 3.3. When restricted to $U(=K \cap(\operatorname{Spin}(10,2) \times S L(2)))$, the irreducible $\left(\mathfrak{e}_{6,0} \oplus \mathfrak{t}_{7,3}\right)$-module $V(n \lambda) \otimes \mathbb{C}_{-4 \xi}$ will decompose as

$$
V(n \lambda) \otimes \mathbb{C}_{-4 \xi}=\bigoplus_{p, q, r \geq 0} V\left(p \omega_{6}+q \omega_{2}\right) \otimes\left(-p-\frac{q}{2}-4\right)_{1} \otimes(-q-2 r-4)_{2},
$$

where $\omega_{6}, \omega_{2}$ are the fundamental weights of Spin(10) corresponding to the simple roots $\alpha_{6}$ and $\alpha_{2}$, and $(\mu)_{i}$ corresponding to the action of $S O(2)_{i}$ as $a \rightarrow a^{\mu}$, where $a \in U(1)$.

Proof. Consider $n=1$ ( $n=0$ is trivial).

We know that $V(\lambda)$ is isomorphic to $\mathfrak{p}^{-}$for they have the same highest weight as $\left(\mathfrak{e}_{6,0} \oplus \mathfrak{t}_{7,3}\right)$-modules. Thus

$$
V(\lambda)=V\left(\omega_{6}\right) \otimes(-1) \otimes(0) \oplus V\left(\omega_{2}\right) \otimes\left(-\frac{1}{2}\right) \otimes(-1) \oplus V_{\text {triv }} \otimes(0) \otimes(-2),
$$

where $V_{\text {triv }}$ is the trivial representation.

The reason is as follows. First, the highest weight vector of weight $\lambda=e_{5}-$ $e_{6}$ must generate an irreducible highest weight representation of $\mathfrak{s o}(10)$, which is $V\left(\omega_{6}\right)$. Second, there is a trivial representation generated by the root vector of the root $\alpha=e_{7}-e_{8}$, which can be read from the action of $\mathfrak{s o}(10)$. Third, another highest 
weight of $\mathfrak{s o}(10)$ occurring in the restriction will be the maximal negative root of the form $\frac{1}{2}\left(e_{7}-e_{8}+\sum_{i}(-1)^{\mu_{i}} e_{i}\right)$ with $\sum_{i} \mu_{i}$ odd, and it is $\frac{1}{2}\left(e_{7}-e_{8}-e_{6}+\sum_{i} e_{i}\right)$. Comparing the dimensions, we can get our conclusion.

For general $n$, using Lemma 3.2, we will have

$$
V(n \lambda) \supseteq \bigoplus_{p, q, r \geq 0} V\left(p \omega_{6}+q \omega_{2}\right) \otimes\left(-p-\frac{q}{2}\right)_{1} \otimes(-q-2 r)_{2} .
$$

Now we want to show that the inclusion above is an equality. We will use induction to show that both sides have the same dimension.

Let $d_{1}(n)=\operatorname{dim} V(n \lambda)$ and

$$
d_{2}(n)=\sum_{p+q \leq n, p, q \geq 0} \operatorname{dim} V\left(p \omega_{6}+q \omega_{2}\right) .
$$

When $n=1$ it is obvious that $d_{1}(1)=d_{2}(1)$. Assuming $d_{1}(n)=d_{2}(n)$ for $n \geq 1$, we want to show that $d_{1}(n+1)=d_{2}(n+1)$. Observe that

$$
c_{2}(n)=d_{2}(n+1)-d_{2}(n)=\sum_{p+q=n+1} \operatorname{dim} V\left(p \omega_{6}+q \omega_{2}\right)
$$

is easier to compute than $d_{2}(n)$. So we turn to show that $c_{1}(n)=d_{1}(n+1)-d_{1}(n)=$ $c_{2}(n)$.

We will use the Weyl dimension formula. Recall the root systems for $\mathfrak{e}_{6,0}$ and $\mathfrak{s o}(10)$ first.

The positive roots for $\mathfrak{e}_{6,0}$ are

$$
\begin{aligned}
& \frac{1}{2}\left(e_{8}-e_{7}-e_{6}+\sum_{i=1}^{5}(-1)^{\nu_{i}} e_{i}\right), \quad \sum_{i} \nu_{i} \quad \text { even, } \\
& e_{i} \pm e_{j}, \quad 1 \leq j<i \leq 5
\end{aligned}
$$

The half sum of the positive roots for $\mathfrak{e}_{6,0}$ is

$$
\rho_{1}=(0,1,2,3,4,-4,-4,4) .
$$

The positive roots for $\mathfrak{s o}(10)$ are

$$
e_{i} \pm e_{j}, \quad 1 \leq j<i \leq 5,
$$

and the half sum of the positive roots is

$$
\rho_{2}=(0,1,2,3,4,0,0,0) .
$$

For convenience, we write

$$
\rho_{2}=(0,1,2,3,4) .
$$

The Weyl dimension formula says that given an irreducible highest weight representation of a complex Lie algebra, if the highest weight is $\lambda$, then the dimension of the representation is

$$
d(\lambda)=\frac{\prod_{\alpha \in \Delta^{+}}(\lambda+\rho, \alpha)}{\prod_{\alpha \in \Delta^{+}}(\rho, \alpha)} .
$$

In our case, $\lambda=e_{5}-e_{6}$, and from the formula, it is enough to consider the roots contributing to the above product. 
For $\mathfrak{e}_{6,0}$, it is enough to consider the following roots:

$$
\begin{aligned}
& \frac{1}{2}\left(e_{8}-e_{7}-e_{6}+e_{5}+\sum_{1 \leq i \leq 4}(-1)^{\mu_{i}} e_{i}\right), \quad \sum_{i} \mu_{i} \text { even, } \\
& e_{5} \pm e_{i}, \quad 1 \leq i \leq 4 .
\end{aligned}
$$

For $\mathfrak{s o}(10)$, it is enough to consider the following roots:

$$
\begin{array}{ll}
e_{5} \pm e_{i}, & 1 \leq i \leq 4, \\
e_{i}+e_{j}, & 1 \leq j<i \leq 4 .
\end{array}
$$

With the above settings, we find

$$
\operatorname{dim} V(n \lambda)=\frac{1}{56}\left(\begin{array}{c}
n+8 \\
5
\end{array}\right)\left(\begin{array}{c}
n+11 \\
11
\end{array}\right)
$$

Noting that $\omega_{6}=(0,0,0,0,1)$ and $\omega_{2}=\frac{1}{2}(1,1,1,1,1)$, we have

$$
\operatorname{dim} V\left(p \omega_{6}+q \omega_{2}\right)=\frac{(q+3)}{105}\left(\begin{array}{c}
p+q+7 \\
4
\end{array}\right)\left(\begin{array}{c}
p+4 \\
4
\end{array}\right)\left(\begin{array}{c}
q+5 \\
5
\end{array}\right) \text {. }
$$

We can easily check the identity

$$
\begin{aligned}
& \frac{1}{56}\left\{\left(\begin{array}{c}
n+9 \\
5
\end{array}\right)\left(\begin{array}{c}
n+12 \\
11
\end{array}\right)-\left(\begin{array}{c}
n+8 \\
5
\end{array}\right)\left(\begin{array}{c}
n+11 \\
11
\end{array}\right)\right\} \\
& =\frac{1}{105}\left(\begin{array}{c}
n+8 \\
4
\end{array}\right) \sum_{q=0}^{n+1}(q+3)\left(\begin{array}{c}
n+5-q \\
4
\end{array}\right)\left(\begin{array}{c}
q+5 \\
5
\end{array}\right),
\end{aligned}
$$

and therefore we have that

$$
\operatorname{dim} V((n+1) \lambda)-\operatorname{dim} V(n \lambda)=\sum_{p+q=n+1} \operatorname{dim} V\left(p \omega_{6}+q \omega_{2}\right),
$$

i.e., $c_{1}(n)=c_{2}(n)$.

\section{Description of the SPeCtrum}

4.1. Preliminaries. The description of the discrete spectrum involves unitary representations with nonzero cohomology which were constructed by Vogan and Zuckerman [28, so it is necessary to give a brief introduction here and fix the notation.

The following results are from [28].

Let $G$ be a real connected semisimple Lie group with finite center, $\mathfrak{g}_{0}$ the Lie algebra of $G$ and $\mathfrak{g}=\left(\mathfrak{g}_{0}\right)_{\mathbb{C}}$ its complexification. Let $K \subset G$ be a maximal compact subgroup, $\theta$ the Cartan involution and

$$
\mathfrak{g}=\mathfrak{k}+\mathfrak{p}
$$

the corresponding Cartan decomposition. Let $i \mathfrak{t}_{0} \in \mathfrak{k}_{0}$ be a Cartan subalgebra of $\mathfrak{k}_{0}$ and fix an element $x$ in $\mathfrak{t}_{0}$. Since $K$ is compact, the linear transformation $\operatorname{ad}(x)$ of $\mathfrak{g}$ is diagonalizable, with real eigenvalues. Define

$$
\begin{aligned}
\mathfrak{q} & =\text { sum of nonnegative eigenspaces of } \operatorname{ad}(x), \\
\mathfrak{u} & =\text { sum of positive eigenspaces of } \operatorname{ad}(x), \\
\mathfrak{l} & =\text { sum of zero eigenspaces of } \operatorname{ad}(x)=\text { centralizer of } x .
\end{aligned}
$$

Then $\mathfrak{q}$ is a parabolic subalgebra of $\mathfrak{g}$, and

$$
\mathfrak{q}=\mathfrak{l}+\mathfrak{u}
$$


is the Levi decomposition. Furthermore, $\mathfrak{l}$ is the complexification of $\mathfrak{l}_{0}=\mathfrak{q} \cap \mathfrak{g}_{0}$. Since $\theta(x)=x$, and $\mathfrak{q}, \mathfrak{l}, \mathfrak{u}$ are all invariant under $\theta$, we have $\mathfrak{q}=\mathfrak{q} \cap \mathfrak{k}+\mathfrak{q} \cap \mathfrak{p}$. Call the subalgebra obtained in this way a $\theta$-stable parabolic subalgebra of $\mathfrak{g}$. Let $K$ and $L$ be the connected subgroups of $G$ with Lie algebras $\mathfrak{t}_{0}$ and $\mathfrak{l}_{0}$ respectively. Let $\Delta(\mathfrak{g})$ denote the roots of $\mathfrak{t}$ in $\mathfrak{g}$. Fix a positive system $\Delta^{+}$of the roots of $\mathfrak{t}$ in $\mathfrak{l}$. Then $\Delta^{+}(\mathfrak{g})=\Delta^{+}(\mathfrak{l}) \cup \Delta(\mathfrak{u})$ is a positive system for $\Delta(\mathfrak{g})$. Now extend $\mathfrak{t}$ to a Cartan subalgebra $\mathfrak{h}$ of $\mathfrak{g}$ and let $\Delta^{+}(\mathfrak{h}, \mathfrak{g})$ be a positive system of roots of $\mathfrak{h}$ in $\mathfrak{g}$ such that its restriction to $\mathfrak{t}$ gives $\Delta^{+}(\mathfrak{g})$. Let $\rho$ denote the half sum of roots in $\Delta^{+}(\mathfrak{h}, \mathfrak{g})$ and $\rho(\mathfrak{u} \cap \mathfrak{p})$ the half sum of roots of $\mathfrak{t}$ in $\mathfrak{u} \cap \mathfrak{p}$. Let $\lambda$ be a one dimensional representation of $\mathfrak{l}$ such that

(1) $\lambda$ is the differential of a unitary character of $L$,

(2) if $\alpha \in \Delta(\mathfrak{u})$, then $\left\langle\alpha,\left.\lambda\right|_{\mathfrak{t}}\right\rangle \geq 0$.

Let $\mu(\mathfrak{q}, \lambda)$ be the representation of $K$ of highest weight $\left.\lambda\right|_{\mathfrak{t}}+2 \rho(\mathfrak{u} \cap \mathfrak{p})$. In [28, Vogan and Zuckerman prove the existence and uniqueness of an irreducible representation $A_{\mathfrak{q}}(\lambda)$ with lowest $K$-type $\mu(\mathfrak{q}, \lambda)$ and infinitesimal character $\lambda+\rho$.

Theorem 4.1 (Vogan and Zuckerman). Assume $\lambda$ is zero on the orthogonal complement of $\mathfrak{t}$ in $\mathfrak{h}$. Let $\pi$ be an irreducible unitary representation of $G$ and assume that:

(a) The $\mathfrak{k}$ representation $\mu(\mathfrak{q}, \lambda)$ occurs in $\pi$.

(b) It has infinitesimal character $\lambda+\rho$.

Then $\pi \simeq A_{\mathfrak{q}}(\lambda)$.

We will see in the next section that most of the representations of $\operatorname{Spin}(10,2)$ lifted from discrete series of $S L(2, \mathbb{R})$ are $A_{\mathfrak{q}}(\lambda)$, and we will give detailed information about them.

4.2. Description. We have determined the restriction of $\pi_{\min }$ to $U$. As the representations of $S L(2, \mathbb{R})$ are well known [27, we can identify the representations from the above restriction directly.

Fixing $p$ and $q$ and from the $K$-type information, we know that the representation of $S L(2, \mathbb{R})$ is the antiholomorphic discrete series representation with highest $K$ type $(-q-4)$. So we get the description of $\pi_{-q-4}$.

For $\theta_{q+4}$, we can read the $K$-types of it from the correspondence

$$
\left.\theta_{q+4}\right|_{S p i n(10) \otimes S O(2)}=\bigoplus_{p \geq 0} V\left(p \omega_{6}+q \omega_{2}\right) \otimes\left(-p-\frac{q}{2}-4\right)_{1} .
$$

Also, since this is the $\alpha$-case in [23], we have a correspondence of infinitesimal characters of $\theta_{k}$ and $\pi_{k}$. This can be read from [11. The infinitesimal character of $\pi_{-q-4}$ is $q+3$. So the infinitesimal character of $\theta_{q+4}$ is

$$
(q+3-5) \omega_{2}+\rho=(q-2) \omega_{2}+\rho,
$$

where $\omega_{2}$ is the fundamental weight of $\alpha_{2}$ in the root system of $\operatorname{Spin}(10,2)$.

Note that the highest weight of the lowest $K$-type of $\theta_{q+4}$ in the sense of Vogan (i.e. $\lambda$ is a highest weight of $V\left(p \omega_{6}+q \omega_{2}\right) \otimes\left(-p-\frac{q}{2}-4\right)_{1}$ so that $\left\langle\lambda+2 \rho_{c}, \lambda+2 \rho_{c}\right\rangle$ is minimal) is

$$
\Lambda_{q}=\left(\frac{q}{2}, \frac{q}{2}, \frac{q}{2}, \frac{q}{2}, \frac{q}{2},-\frac{q}{2}-4\right)
$$


(i.e., $p=0$ ). Now that we have determined the $K$-type structure and infinitesimal character of $\theta_{k}$, we can almost identify the representation. It remains to describe it.

Let $x_{0}=(1,1,1,1,1,-1)$ in the standard coordinate system of the root system of $\mathfrak{s o}(12)$, and let $\mathfrak{q}=\mathfrak{q}\left(x_{0}\right)$ be the parabolic subalgebra of $\mathfrak{s o}(12)$ defined as the sum of the eigenspaces for $\operatorname{ad}\left(x_{0}\right)$ with nonnegative eigenvalues.

Let $\mathfrak{q}=\mathfrak{l}+\mathfrak{u}$, where $\mathfrak{l}$ is the Levi component and $\mathfrak{u}$ is the nilradical of $\mathfrak{q}$. From the definition, we know that $\mathfrak{l}$ is the sum of zero eigenspaces of $\operatorname{ad}\left(x_{0}\right)$ and $\mathfrak{u}$ is the sum of positive eigenspaces of $\operatorname{ad}\left(x_{0}\right)$.

For $\lambda=(q-2) \omega_{2}$, define the representation $R_{\mathfrak{q}}^{S}(\lambda)=R_{\mathfrak{q}}^{S}\left((q-2) \omega_{2}\right)$ as in [21]. The parameter $(q-2) \omega_{2}$ is always in the weakly fair range. So $R_{\mathfrak{q}}^{j}\left((q-2) \omega_{2}\right)=0$ unless $j=S=\operatorname{dim}(\mathfrak{u} \cap \mathfrak{k})=10$.

Proposition 4.1. For $q \geq 0$, the representation $\theta_{q+4}$ and $R_{\mathfrak{q}}^{S}\left((q-2) \omega_{2}\right)$ have the same infinitesimal character and $K$-type structure.

Proof. Let $\mathfrak{q}=\mathfrak{l} \oplus \mathfrak{u}$, where $\mathfrak{q}$ is the parabolic subalgebra defined above. We know that $\mathfrak{l}$ is the centralizer of $x_{0}$ and $\mathfrak{u}$ is the sum of the eigenspaces for $\operatorname{ad}\left(x_{0}\right)$ with positive eigenvalues.

It is easy to see that $[\mathfrak{l}, \mathfrak{l}]$ is of type $A_{5}$, and it is in fact $\mathfrak{s u}(5,1)$.

We will consider a set of simple roots for $\mathfrak{s o}(10,2)$ such that all roots in $\mathfrak{u}$ are positive for the system determined by the simple roots. Let the first five roots $\left\{\alpha_{2}, \alpha_{3}, \alpha_{4}, \alpha_{5}, \alpha_{6}\right\}$ be unchanged and take $\alpha_{7}^{\prime}=-e_{6}-e_{5}$. These together form a system of simple roots for $\mathfrak{s o}(10,2)$. With respect to this system, we can easily see that $x_{0}$ is the fundamental weight of $\alpha_{2}$ and the Levi decomposition of $\mathfrak{q}$ can be easily read from this.

The representation $R_{\mathfrak{q}}^{S}\left((q-2) \omega_{2}\right)$ has infinitesimal character $(q-2) \omega_{2}+\rho$, which is the same as $\theta_{q+4}$.

Also, we can see that $\Lambda_{q}=(q-2) \omega_{2}+2 \rho(\mathfrak{u} \cap \mathfrak{p})=\left(\frac{q}{2}, \frac{q}{2}, \frac{q}{2}, \frac{q}{2}, \frac{q}{2},-\frac{q}{2}-4\right)$, which is the highest weight of the lowest $K$-type specified above.

Let $\phi$ be an irreducible representation of $K$ which acts on the space $\mathbf{Z}$. The weight $\Lambda_{q}$ defines a one dimensional representation of $L \cap K$. Let

$$
S(\mathfrak{u} \cap \mathfrak{p})=\sum_{n=0}^{\infty} S^{n}(\mathfrak{u} \cap \mathfrak{p})
$$

be the symmetric algebra of $\mathfrak{u} \cap \mathfrak{p}$. The proof of the generalized Blattner formula (in [9] ) shows that the multiplicity of $\phi$ in $R_{\mathfrak{q}}^{S}\left((q-2) \omega_{2}\right)$ is equal to the dimension of the space

$$
H_{L} m_{L \cap K}\left(H^{0}(\mathfrak{u} \cap \mathfrak{k}, Z), S(\mathfrak{u} \cap \mathfrak{p}) \otimes \Lambda_{q}\right) .
$$

Since the highest weight vectors of the $L \cap K$-module $H^{0}(\mathfrak{u} \cap \mathfrak{k}, \mathbf{Z})$ are precisely the highest weight vectors of the $K$-module $\mathbf{Z}$, it is sufficient to decompose $S^{n}(\mathfrak{u} \cap \mathfrak{p}), n=$ $0,1,2, \ldots$ as $L \cap K$-modules.

It is easy to check that the semisimple part of $\mathfrak{l} \cap \mathfrak{k}$ is $\mathfrak{s u}(5)$ generated by the simple roots $\left\{\alpha_{3}, \alpha_{4}, \alpha_{5}, \alpha_{6}\right\}$. Letting its center be $\eta$, it acts on $\mathfrak{u} \cap \mathfrak{p}$ by $e_{5}-e_{6}$, since $\mathfrak{u} \cap \mathfrak{p}$ is an irreducible module of $\mathfrak{l} \cap \mathfrak{k}$, and as a $\mathfrak{l} \cap \mathfrak{k}$-module,

$$
\mathfrak{u} \cap \mathfrak{p}=V\left(e_{5}-e_{6}\right),
$$


where $V\left(e_{5}-e_{6}\right)$ is the highest weight module of weight $e_{5}-e_{6}$. So we obtain

$$
S(\mathfrak{u} \cap \mathfrak{p}) \otimes \Lambda_{q}=\bigoplus_{n \geq 0}\left(\frac{q}{2}, \frac{q}{2}, \frac{q}{2}, \frac{q}{2}, n+\frac{q}{2},-\frac{q}{2}-n-4\right),
$$

which are precisely the $K$-types of $\theta_{q+4}$.

With respect to the positive root system defined in the proof of the above proposition, we have

$$
\rho=(0,1,2,3,4,-5) \text {. }
$$

It is easy to check that $(q-2) \omega_{2}$ is always in the fair range. For $q \geq 1$, it is in the weakly good range, so $R_{\mathfrak{q}}^{S}\left((q-2) \omega_{2}\right)$ is irreducible. Finally, if $q \geq 2$, then $R_{\mathfrak{q}}^{S}\left((q-2) \omega_{2}\right)=A_{\mathfrak{q}}\left((q-2) \omega_{2}\right)$ is a unitary representation with nonzero cohomology, and in this case, the infinitesimal character and the full $K$-type structure can determine the isomorphic class of the representation. So the above proposition implies:

Theorem 4.2. For $q \geq 2$, we have $\theta_{q+4} \simeq A_{\mathfrak{q}}\left((q-2) \omega_{2}\right)$.

Noting that $\operatorname{dim}\left(\mathfrak{u} \cap \mathfrak{p}^{+}\right)=0$ and $\operatorname{dim}\left(\mathfrak{u} \cap \mathfrak{p}^{-}\right)=5$, the representation $A_{\mathfrak{q}}((q-$ 2) $\omega_{2}$ ) has nonzero cohomology in bi-degree $(0,5)$. In fact, from the coordinate of $x_{0}$, we know in [10] that the only representations with nonzero cohomology of degree 5 are of bi-degree $(0,5)$ and $(5,0)$. Up to conjugation, there are 4 families of unitary representations with nonzero cohomology of degree 5 .

From [10] and [17, the classical theta lifting can only produce unitary representations with nonzero cohomology of bi-degree $(r, r)(r \leq 10)$. So our lifting gives representations different from classical theta lifting.

Theorem 4.3. $\theta_{q+4}$ for $q \geq 0$ are unitary highest weight modules.

Suppose the Cartan decomposition of $\mathfrak{g}=\mathfrak{s o}(10,2)$ is $\mathfrak{g}=\mathfrak{k} \oplus \mathfrak{p}^{+} \oplus \mathfrak{p}^{-}$. Let $\mathfrak{h} \in \mathfrak{k}$ be a Cartan subalgebra of $\mathfrak{g}$. We know that the $K$-type decomposition of $\theta_{q+4}$ is

$$
\left.\theta_{q+4}\right|_{\operatorname{Spin}(10) \otimes S O(2)}=\bigoplus_{p \geq 0} V\left(p \omega_{6}+q \omega_{2}\right) \otimes\left(-p-\frac{q}{2}-4\right)_{1} .
$$

The lowest $K$-type is $V\left(q \omega_{2}\right) \otimes\left(-\frac{q}{2}-4\right)_{1}$. Letting $v$ be a vector in $V\left(q \omega_{2}\right) \otimes\left(-\frac{q}{2}-4\right)_{1}$, we want to show that for any root vector $X \in \mathfrak{p}^{+}, X \cdot v=0$.

Let $t \in \mathfrak{h}$. Then

$$
t \cdot X \cdot v=[t, X] \cdot v+X \cdot t \cdot v .
$$

As $X \in \mathfrak{p}^{+}$is a root vector, $[t, X]=\alpha(t) X$ for some noncompact positive root $\alpha$. Also, as $v \in V\left(q \omega_{2}\right) \otimes\left(-\frac{q}{2}-4\right)_{1}, t \cdot v=\chi(t) v$ for some character $\chi$. So we can get

$$
t \cdot X \cdot v=(\alpha(t)+\chi(t)) X \cdot v .
$$

Note that the positive noncompact roots for $\mathfrak{s o}(10,2)$ are $e_{6} \pm e_{i}$ for $1 \leq i \leq 5$, so the ()$_{1}$ component of $\alpha+\chi$ will be $1-\frac{q}{2}-4$. However, from our description of $K$-types of $\theta_{q+4}$ we know that no such vector occurs. So we must have $X \cdot v=0$.

Next, we want to show that this is an irreducible representation; i.e., the action of $\mathfrak{p}^{-}$on $V\left(q \omega_{2}\right) \otimes\left(-\frac{q}{2}-4\right)_{1}$ will generate the full space of $K$-finite vectors. Note that the roots in $\mathfrak{p}^{-}$are of the form $e_{i} \pm e_{6}$ and the highest root is $\beta=e_{5}-e_{6}$. Taking a root vector $X \in \mathfrak{g}_{\beta}$, we know that the action of $X$ on the highest weight vector $v \in$ $V\left(q \omega_{2}\right) \otimes\left(-\frac{q}{2}-4\right)_{1}$ is a highest weight vector of the $K$-type $V\left(\omega_{6}+q \omega_{2}\right) \otimes\left(-1-\frac{q}{2}-4\right)$. 
So if we repeat this process with $X \in \mathfrak{p}^{-}$, we can get the full $K$-finite vector space. This shows that the representation $\theta_{q+4}$ is irreducible.

\section{ACKNOWLEDGEMENTS}

This paper is the first part of the author's doctoral dissertation at HKUST. The author would like to thank his thesis advisor, Professor Jian-Shu Li, for his guidance and constant encouragement and for many enlightening and helpful discussions. He also thanks the referee for helpful comments and suggestions.

\section{REFERENCES}

1. Bourbaki, Nicolas, Lie groups and Lie algebras, Chapters 4-6, translated from the 1968 French original by Andrew Pressley. Elements of Mathematics (Berlin), Springer-Verlag, Berlin, 2002. MR.1890629 (2003a:17001)

2. Collingwood, David H.; McGovern, William M., Nilpotent orbits in semisimple Lie algebras. Van Nostrand Reinhold Mathematics Series. Van Nostrand Reinhold Co., New York, 1993. MR 1251060 (94j:17001)

3. Enright, Thomas; Howe, Roger; Wallach, Nolan, A classification of unitary highest weight modules. Representation theory of reductive groups (Park City, Utah, 1982), 97-143, Progr. Math., 40, Birkhäuser Boston, Boston, MA, 1983. MR733809 (86c:22028)

4. Howe, Roger, $\theta$-series and invariant theory. Automorphic forms, representations and $L$ functions (Proc. Sympos. Pure Math., Oregon State Univ., Corvallis, Ore., 1977), Part 1, pp. 275-285, Proc. Sympos. Pure Math., XXXIII, Amer. Math. Soc., Providence, RI, 1979. MR 546602 (81f:22034)

5. Howe, Roger, Reciprocity laws in the theory of dual pairs. Representation theory of reductive groups (Park City, Utah, 1982), 159-175, Progr. Math., 40, Birkhäuser Boston, Boston, MA, 1983. MR $733812(85 \mathrm{k}: 22033)$

6. Howe, Roger, Transcending classical invariant theory. J. Amer. Math. Soc. 2 (1989), No. 3, 535-552. MR985172 (90k:22016)

7. Howe, Roger, Remarks on classical invariant theory. Trans. Amer. Math. Soc. 313 (1989), No. 2, 539-570. MR986027 (90h:22015a)

8. Huang, Jing-Song; Pandzic, Palve; Sacin, Gordan, New dual pair correspondence. Duke Math. J. 82 (1996), No. 2, 447-471. MR1387237 (97c:22015)

9. Hecht, Henryk; Schmid, Wilfried, A proof of Blattner's conjecture. Invent. Math. 31 (1975), No. 2, 129-154. MR0396855 (53:715)

10. Harris, Michael; Li, Jian-Shu, A Lefschetz property for subvarieties of Shimura varieties. J. Algebraic Geom. 7 (1998), No. 1, 77-122. MR1620690 (99e:14027)

11. Li, Jian-Shu, The correspondence of infinitesimal characters for reductive dual pairs in simple Lie groups, Duke Mathematics Journal 97 (1999), No. 2, 347-377. MR.1682229 (2000b:22014)

12. Li, Jian-Shu, A description of the discrete spectrum of $\left(S L(2), E_{7(-25)}\right)$, Asian J. Math. 3 (1999), No. 2, 359-372. MR1796508 (2001m:22029)

13. Li, Jian-Shu, Minimal representations and reductive dual pairs. Representation theory of Lie groups (Park City, UT, 1998), 293-340, IAS/Park City Math. Ser., 8, Amer. Math. Soc., Providence, RI, 2000. MR1737731 (2001a:22013)

14. Li, Jian-Shu, Two reductive dual pairs in groups of type E. Manuscripta Math. 91 (1996), No. 2, 163-177. MR1411651 (97j:22037)

15. Li, Jian-Shu, Singular unitary representations of classical groups. Invent. Math. 97 (1989), No. 2, 237-255. MR1001840(90h:22021)

16. Li, Jian-Shu, Theta series and construction of automorphic forms. Representation theory of groups and algebras, 237-248, Contemp. Math., 145, Amer. Math. Soc., Providence, RI, 1993. MR 1216192

17. Li, Jian-Shu, Theta lifting for unitary representations with nonzero cohomology. Duke Math. J. 61 (1990), No. 3, 913-937. MR1084465 (92f:22024)

18. Kudla, Stephen S., Seesaw dual reductive pairs. Automorphic forms of several variables (Katata, 1983), 244-268, Progr. Math., 46, Birkhäuser Boston, Boston, MA, 1984. MR763017 (86b:22032) 
19. Knapp, Anthony W., Lie groups beyond an introduction. Second edition. Progress in Mathematics, 140. Birkhäuser Boston, Inc., Boston, MA, 2002. MR.1920389 (2003c:22001)

20. Knapp, Anthony W. Representation theory of semisimple groups. An overview based on examples. Reprint of the 1986 original. Princeton Landmarks in Mathematics. Princeton University Press, Princeton, NJ, 2001. MR.1880691 (2002k:22011)

21. Knapp, Anthony W.; Vogan, David A., Jr., Cohomological induction and unitary representations. Princeton Mathematical Series, 45. Princeton University Press, Princeton, NJ, 1995. MR:1330919 (96c:22023)

22. Kobayashi, Toshiyuki, Multiplicity-free theorems of the restrictions of unitary highest weight modules with respect to reductive symmetric pairs. Representation theory and automorphic forms, 45-109, Progr. Math., 255, Birkhäuser Boston, Boston, MA, 2008. MR2369496 $(2008 \mathrm{~m}: 22024)$

23. Rallis, S.; Schiffmann, G., The orbit and theta correspondences for some dual pairs. J. Math. Kyoto Univ. 35 (1995), 429-493. MR1359007 (96k:22027)

24. Vogan, David A., Jr., Gelfand-Kirillov dimension for Harish-Chandra modules. Invent. Math. 48 (1978), No. 1, 75-98. MR0506503 (58:22205)

25. Vogan, David A., Jr., The algebraic structure of the representation of semisimple Lie groups. I. Ann. of Math. (2) 109 (1979), No. 1, 1-60. MR519352 (81j:22020)

26. Vogan, David A., Jr., Singular unitary representations. Noncommutative harmonic analysis and Lie groups (Marseille, 1980), pp. 506-535, Lecture Notes in Math., 880, Springer, BerlinNew York, 1981. MR644845 (83k:22036)

27. Vogan, David A., Jr., Representations of real reductive Lie groups. Progress in Mathematics, 15. Birkhäuser, Boston, Mass., 1981. MR632407 (83c:22022)

28. Vogan, David A., Jr.; Zuckerman, Gregg J., Unitary representations with nonzero cohomology. Compositio Math. 53 (1984), No. 1, 51-90. MR762307|(86k:22040)|

Academy of Mathematics and Systems Science, Chinese Academy of Sciences, Beijing, 100190, People's Republic of China

E-mail address: duzhe@amss.ac.cn 